\title{
Glass Transition Temperature of a Polymer Thin Film: Statistical and Fitting Uncertainties
}

\author{
David McKechnie ${ }^{1}$, Jordan Cree ${ }^{1}$, Dominic Wadkin-Snaith ${ }^{2}$, and Karen Johnston ${ }^{1}$ \\ ${ }^{1}$ Department of Chemical and Process Engineering, James Weir Building, University of \\ Strathclyde, 75 Montrose Street, Glasgow G1 1XJ, United Kingdom \\ ${ }^{2}$ Solvay Composite Materials, The Wilton Centre, Redcar, TS10 4RF
}

March 26, 2020

\begin{abstract}
The polymer glass transition is an important property in a wide variety of applications. The glass transition temperature of a polymer composite or confined thin film can be significantly different to the pure polymer. Molecular dynamics simulations are useful for providing molecular level insight and prediction, particularly at interfaces, that are not easily observable experimentally. However, there are significant methodological uncertainties in calculating the polymer glass transition temperature using molecular dynamics simulations. In this work we investigate how the cooling method, fitting range and statistical variation affects the calculated glass transition temperature of polyethylene. We found that it is necessary to perform multiple independent simulations to obtain statistically significant results, and that appropriate fitting ranges must be chosen. The methodological findings were used to investigate the difference in glass transition temperature between pure polyethylene and a polyethylene film confined between graphene surfaces. It was found that the glass transition temperature of a $9 \mathrm{~nm}$ thick confined film was higher than bulk polyethylene by approximately $15 \mathrm{~K}$.
\end{abstract}

\section{Introduction}

Polymer composites are used in a wide variety of applications that take advantage of their mechanical, thermal and electrical properties. The properties of a polymer composite depend strongly on the properties of the polymer-filler interface, and it is therefore desirable to understand the structure and dynamical properties near the interface. One of the important composite properties is the glass transition temperature, $T_{g}$, below which the polymer exhibits hard and relatively brittle glassy properties. The effect of an interface on the $T_{g}$ of a polymer thin film has been a source of controversy in literature, with experiments predicting a wide range of shifts in $T_{g}$ for polystyrene thin films $[1,2]$. Experimentally, it is challenging to probe the interface, as spectroscopic measurements are often dominated by the signal from the bulk material and measurements can be affected by the presence of impurities or defects.

Classical molecular dynamics (MD) simulations can provide insight into the structure and dynamics near the interface, including the glass transition temperature of polymers and thin films and a number of MD studies have studied glassy polymers [3]. There have also been several studies of how surfaces affect $T_{g}$ of a thin polymer film. Free-standing polystyrene (PS) thin films, were found to have a lower $T_{g}$ at the boundary layer compared to the centre of the film $[4,5]$, which is in qualitative agreement with an earlier study by Morita et al who also found that $T_{g}$ of a polymer is lower in the boundary layer than in the bulk [6]. For supported polymer films, a study found that weak interactions with the substrate lowered the polymer $T_{g}$ whereas strong interactions increased $T_{g}[7]$. A study of 
PS at weakly and strongly attractive structureless surfaces found that $T_{g}$ is $20-50 \mathrm{~K}$ higher near the solid surface depending on the strength of polymer-surface attraction [8]. These findings are supported by another study that found that relaxation times were shorter for weak substrate interactions [9], indicating a lower $T_{g}$, and longer for strong interactions. In general, for simulations with structured surfaces, polymer dynamics near an attractive solid surface are slower than in the bulk $[10,11,12]$, which indicates an increase in $T_{g}$.

While simulations have provided an understanding of how a substrate affects the glass transition temperature, challenges remain in quantifying the change in $T_{g}$ due to a surface, in part due to the variation in surface models used as discussed above, but also due to the methodology for obtaining the glass transition temperature in simulations. The methodological details for calculating $T_{g}$ are rarely discussed, but here we show that they can have a significant impact on the results. In simulations $T_{g}$ is usually determined by cooling an equilibrated polymer melt at a specified cooling rate, $\Gamma$, and measuring where the density vs temperature undergoes a gradient change, as shown in Fig. 1(b). $T_{g}$ is found by a linear fit to the fluid and glassy branches of the density-temperature curves as shown in Fig. 1(b). However, there are several variations and uncertainties in the calculation of $T_{g}$. First, $T_{g}$ is known to depend on the cooling rate $[13,14]$, and in simulations the cooling rates are many orders of magnitude faster than experimental cooling rates, and to obtain a reasonable estimate for $T_{g}$ results for a series of fast cooling rates must be extrapolated to slow cooling rates. Second, cooling is usually simulated using either linear [13] or stepped cooling $[8,13]$ as illustrated in Fig. 1(a). Under linear cooling the temperature is gradually reduced at a constant rate, while stepped cooling reduces the temperature in a stepwise fashion with time between the steps of constant temperature simulation to allow for equilibration. Third, $T_{g}$ is sensitive to the ranges used for fitting the fluid and glassy regimes $[15,5]$. Finally, there is sig-
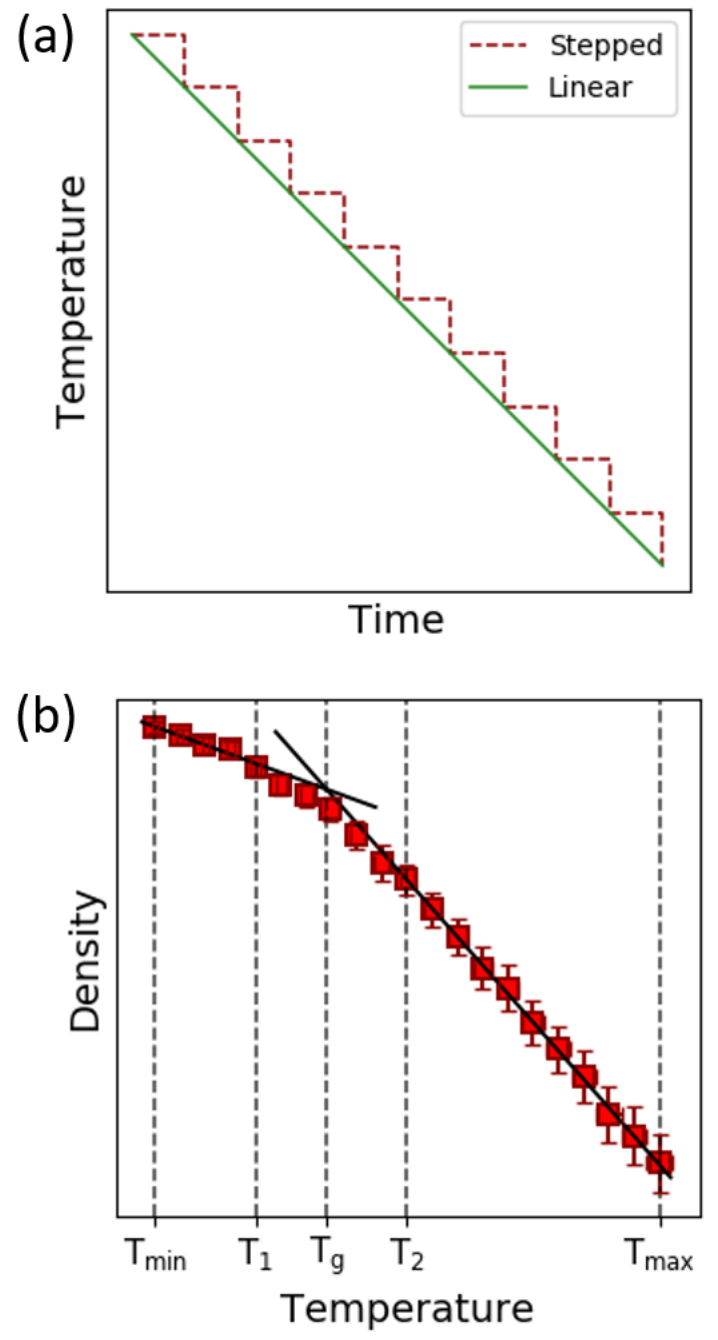

Figure 1: (a) Schematic showing different cooling methods: linear (green solid line) and stepped (red dashed line) (b) An example of the fitting method of density vs temperature curves used to determine $T_{g}$.

nificant statistical variation in simulations and a large number of independent simulations are required [15].

The cooling technique and fitting ranges used are often unreported in literature, and most studies do not mention if they have performed more than a single independent simulation at a particular cooling rate. To obtain a quantitive estimate of the shift in $T_{g}$ at a surface, it is essential to thoroughly test the sensitivity of $T_{g}$ with the 
above mentioned effects. In this study we investigate the shift in $T_{g}$ in a polyethylene $(\mathrm{PE})$ thin film confined between graphene surfaces, carefully accounting for the effect of stepped vs linear cooling techniques, fitting range, and statistical variation.

\section{Methodology}

Polyethylene (PE) chains were modelled using a version of the Paul-Yoon-Smith (PYS) united atom (UA) force field, which was developed for long chain alkanes [16] and treats the $\mathrm{CH}_{2}$ and $\mathrm{CH}_{3}$ groups (referred to as 'beads' or 'monomers') as identical. Bond stretches are represented by harmonic potentials using the parameters from Chang et al [17]. The dihedral potential parameters were taken from Ramos et al [14]. Graphene sheets were modelled with uncharged, fixed atoms using LJ parameters from the DREIDING force field [18]. Potential forms and parameters for polyethylene and graphene are reported in the Supporting Information.

All MD simulations were performed using the LAMMPS software [19]. The simulations were prepared by first running an isolated 50 bead chain in vacuum for a few picoseconds to allow the polymer to coil. This was performed three times and the resulting polymers were used to generate starting configurations. Two different systems have been investigated: a bulk PE melt, and a thin PE film between sheets of graphene.

For the bulk simulations 30 of the pre-coiled chains (10 of each initial structure) were randomly inserted within a simulation box $(20 \times$ $20 \times 20 \mathrm{~nm}$ ). Periodic boundary conditions were applied in all directions to provide bulk-like behaviour. The system was energy minimised to prevent any overlapping atoms and 100 ps of NVT simulation was performed. This was followed by 30 ns of NPT simulation for equilibration. This equilibration time was determined to be sufficient through analysing the density, the radius of gyration, $R_{g}$, and the end-to-end distance, $R_{e}$, to find where they reached their equilibrium values. This procedure was repeated to produce 100 independent starting configurations.

For the surface a similar procedure was performed. A graphene sheet was placed in the $x y$ plane of the simulation box $(3.19 \times 3.40 \times 10 \mathrm{~nm})$ and 60 precoiled chains (20 of each initial structure) were inserted randomly to the simulation box as before. In this instance 60 chains were added to ensure the resulting film would be thick enough to return to a bulk-like region within the centre. Periodic boundaries were applied in all directions to give a film of PE confined between graphene sheets. An energy minimisation was performed and the system was then simulated for 10 ps of NVT simulation, followed by 10 ns of NPT simulation for equilibration. During the NPT simulation the box was only allowed to adjust in the $\mathrm{z}$ direction in order to maintain the size of the graphene sheet. This procedure was repeated to produce 20 independent starting configurations. This resulted in film thicknesses of approximately $9 \mathrm{~nm}$ at $500 \mathrm{~K}$.

Cooling simulations were performed using stepped and linear cooling at cooling rates ranging from $\Gamma=0.005-0.9 \mathrm{~K} \mathrm{ps}^{-1}$. In both cases temperature and pressure were controlled using a Nose-Hoover thermostat and barostat with damping parameters of 0.1 and 1 ps respectively. In the case of linear cooling the temperature was ramped down linearly from 500 to $100 \mathrm{~K}$ over the length of time necessary to achieve the desired cooling rate. During stepped cooling the temperature was reduced in $20 \mathrm{~K}$ steps from 500 to $100 \mathrm{~K}$, with step duration adjusted to reach the desired cooling rate.

In simulations of the confined thin film the carbon atoms of the graphene sheet are fixed in place by holding them at $0 \mathrm{~K}$. This has implications on the temperature of the PE chains compared to the overall system temperature that is being controlled by the thermostat. Therefore the density/temperature curves used to determine the glass transition of the PE film is using 
the temperatures calculated for the PE chains directly, and not of the overall system. The cooling rate obtained by dividing the difference in the initial and final temperatures by the total simulation time is slightly different than those performed from the bulk and the data has been plotted appropriately.

\section{Results and Discussion}

We first present structural properties for PE in bulk and thin film. We next investigate the effect of statistics, cooling method, and fitting range on $T_{g}$ for bulk PE. Finally, we apply the optimal technique to a $\mathrm{PE}$ thin film confined between two graphene surfaces.

\subsection{Properties of PE thin films}

Bulk properties for bulk PE including density and $R_{g}$ were calculated and found to be in good agreement with previous simulations studies. We obtained a density of $0.755 \mathrm{~g} \mathrm{~cm}^{-3}$ for a system with 50 -monomer chains at $500 \mathrm{~K}$, which is slightly higher than the density of $0.730 \mathrm{~g} \mathrm{~cm}^{-3}$ for 50-monomer chains at $509 \mathrm{~K}$ obtained by Hur et al [20]. Ramos et al obtained $0.795 \mathrm{~g} \mathrm{~cm}^{-3}$ for longer 192-monomer chains at a temperature of $500 \mathrm{~K}$. For 50-monomer PE chains, we obtained an $R_{g}$ of $1.03 \mathrm{~nm}$ at $500 \mathrm{~K}$ in comparison to $1.04 \mathrm{~nm}$ obtained by Hur et al [20]. A comparison of $R_{g}$ for different chain lengths is given in Supporting Information.

Fig. 2 shows a snapshot of the confined film alongside structural properties, which have been symmetrised across the film and analysed as a function of distance, $z$, from the graphene surface. A layered structure near the graphene surface can been seen from the snapshot of the system and the monomer density profile in Fig. 2(a) and (b). The high density peaks become smaller until the monomer density reaches the bulk density in the centre of the film. This layering is a typical feature for polymers at solid surfaces $[21,22,23,9,11,24,25,12]$. Fig. 2(c) shows

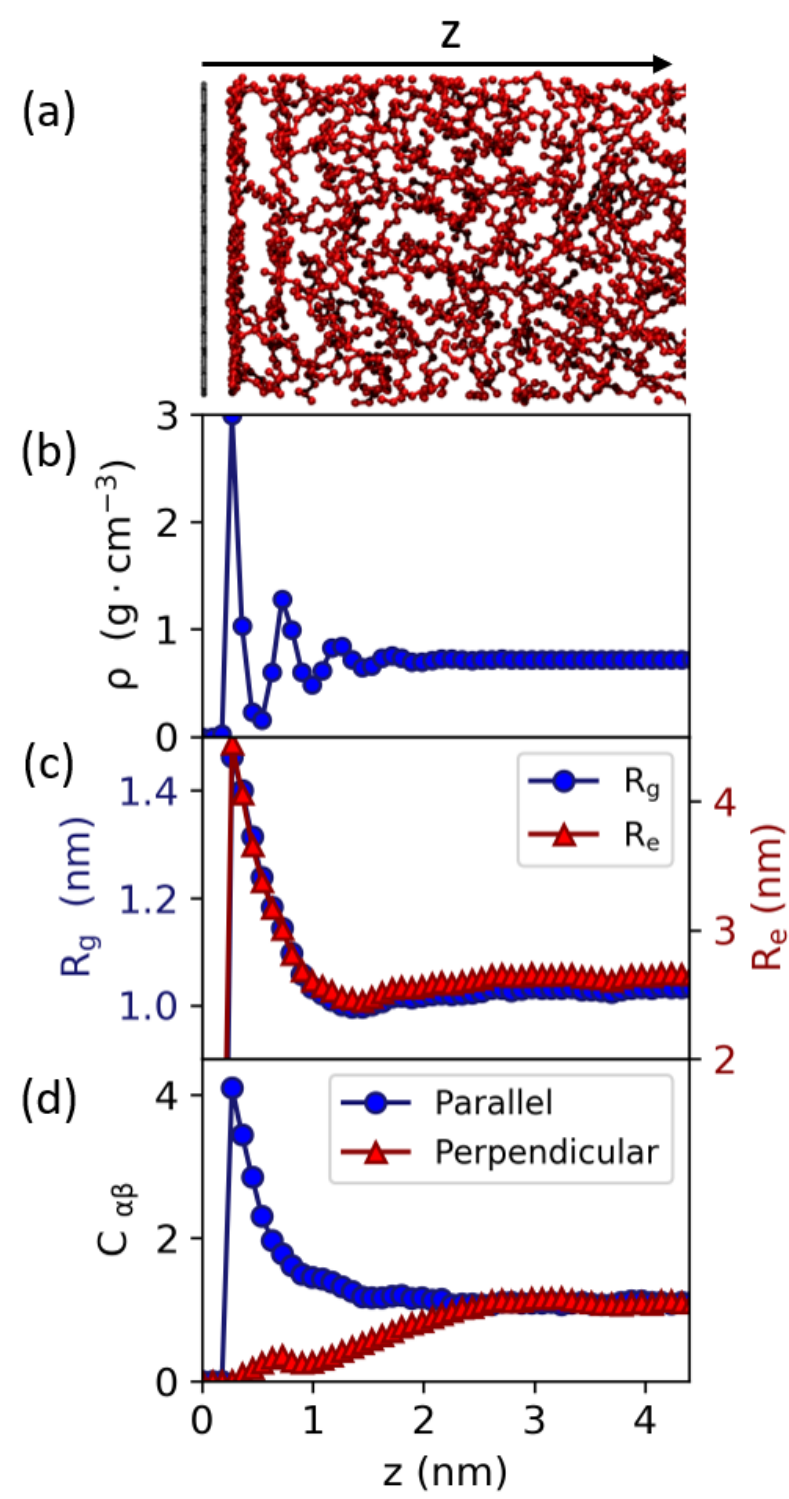

Figure 2: (a) Snapshot of PE film near a graphene surface. The graphs show variation of (b) density, (c) $R_{g}$ and $R_{e}$, and (d) conformation tensor, with distance $z$ from the graphene surface.

$R_{g}$ and $R_{e}$ vs distance for the thin film. Near the surface $R_{g}$ and $R_{e}$ are significantly higher than their bulk values of $1.03 \mathrm{~nm}$ and $2.68 \mathrm{~nm}$, respectively. They reach their bulk values after approximately $3 \mathrm{~nm}$.

The conformation tensor, shown in Fig. 2(d), gives information about the orientation of the 
polymer chains. The conformation tensor is defined as:

$$
C_{\alpha \beta}=3 \frac{\left\langle R_{e \alpha} R_{e \beta}\right\rangle}{\left\langle R_{e, b u l k}^{2}\right\rangle}
$$

where $\alpha$ and $\beta$ are Cartesian components of the $R_{e}$ vector. For the bulk polymer with randomly oriented chains, $C_{\alpha \alpha}=1$ and $C_{\alpha \beta}=0$. For the thin film the off-diagonal terms will be zero and the diagonal terms $C_{\alpha \alpha}$ will no longer equal unity but we expect $C_{x x}$ and $C_{y y}$ to be equal as there is no symmetry breaking parallel to the surface plane. We therefore consider the $C_{z z}$ element, which is perpendicular to the graphene surface, and $C_{\text {par }}=\frac{1}{2}\left(C_{x x}+C_{y y}\right)$ which is parallel to the graphene surface. The conformation tensor profile shows that near the graphene surface the polymer chains stretch out parallel to the surface and flatten in the $z$-direction. This stretching of the polymer chains along the interface has been previously seen in a study of a polystyrene-gold interface [26].

\subsection{Methodology for calculating the glass transition temperature}

\subsubsection{Statistical variation}

We start our investigations of $T_{g}$ by looking at the statistical variation in independent simulations. To quantify the variation, 100 independent simulations were run using stepped cooling at a cooling rate $0.9 \mathrm{~K} \mathrm{ps}^{-1}$. This is the highest cooling rate investigated in this work, and is therefore expected to have the largest variability. To estimate $T_{g}$ the fitting ranges were fixed at $100-200 \mathrm{~K}$ for the glassy (low $T$ ) branch, and 300-500 K for the fluid (high $T$ ) branch.

The running average of $T_{g}$ from these independent simulations is shown in Fig. 3, reaching an average value of $238 \mathrm{~K}$ over all 100 simulations. The running average decreases with the number of simulations due to the first simulation having a very high value of $T_{g}$, and the $T_{g}$ values show a Gaussian distribution (see Supporting Information. The minimum and maximum values of $T_{g}$ were $189 \mathrm{~K}$ and $300 \mathrm{~K}$, highlighting the importance in performing multiple independent simulations. Performing just one simulation in this instance could result in a value for $T_{g}$ that is over $60 \mathrm{~K}$ from what would be obtained from averaging across a large number of simulations.

To determine how the error in the obtained $T_{g}$ varied with the number of simulations performed statistical bootstrapping was performed. From the 100 values obtained for $T_{g}$ a random sample of $N$ simulations was selected, with nothing preventing a given value being selected multiple times, and the average of this sample was taken. This process was repeated 100 times and the standard deviation of the average values was taken as the error after $N$ simulations was completed. The results of the bootstrapping are shown in Fig. 3. After 20 simulations the error was reduced to below $5 \mathrm{~K}$, and we decided to use 20 simulations for all cooling rates.

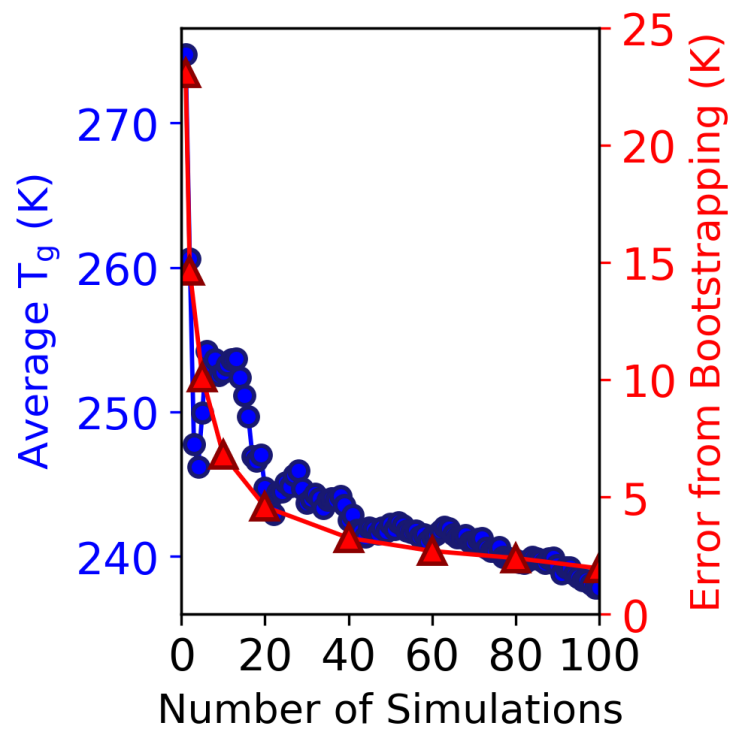

Figure 3: Running average (circles, primary axis) and error (triangles, secondary axis) of $T_{g}$ with increasing number of simulations at $0.9 \mathrm{~K} \mathrm{ps}^{-1}$.

It has been reported that there is a large statistical variability in the glass transition temperature obtained by simulation. In a study of a generic bead spring polymer model it was found 
that there was still significant statistical errors in the obtained $T_{g}$ after 55 independent simulations [15]. It has previously been observed that for very thin films that exhibit slow glasslike dynamics, there can be a large variation in film properties, such as density and $R_{e}$ [11], and therefore averaging over several independent simulations provides improved sampling compared to extending simulation times.

\subsubsection{Cooling technique}

We investigate the effect of linear vs stepped cooling (as described in Section 2) on $T_{g}$. Eight cooling rates ranging from 0.005 up to $0.9 \mathrm{~K} \mathrm{ps}^{-1}$ were simulated, and 20 independent simulations were performed at each cooling rate. The results are shown in Figure 4. The fitting ranges were set to 100-200 K for the glassy branch and 300$500 \mathrm{~K}$ for the fluid branch.

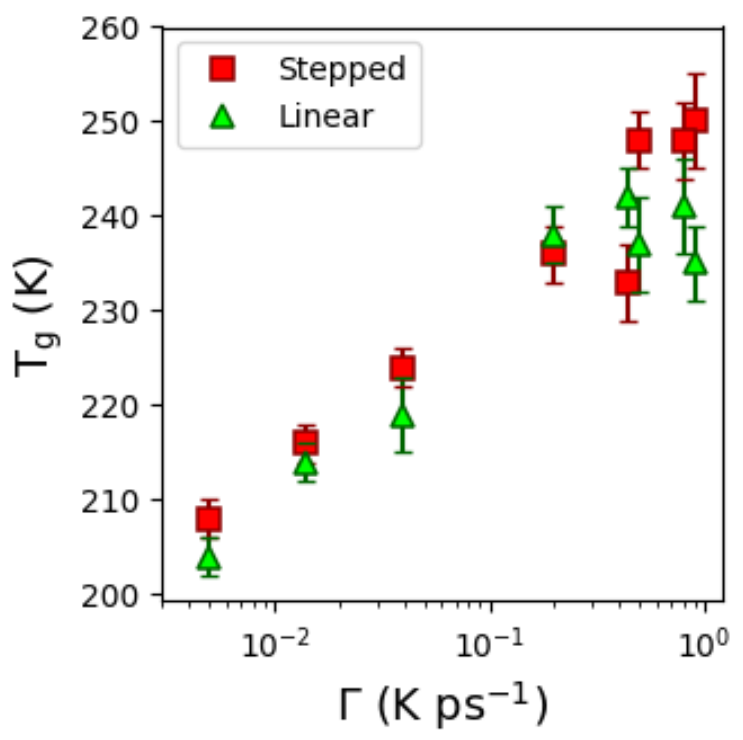

Figure 4: $T_{g}$ vs cooling rate for stepped (red squares) and linear (green triangles) cooling methods. Error bars show the standard error over the 20 independent simulations.

As can be seen there is good agreement between the two cooling methods, particularly for slower cooling. At the lower cooling rates investigate the values all fall within the standard error between the two methods, however at the higher cooling rates the values obtained using stepped cooling fall higher than those obtained using linear cooling. Using linear cooling also results in a higher uncertainty in the obtained glass transition temperature and so stepped cooling was used for the remaining $T_{g}$ simulations.

We note that an alternative method, called sampled cooling has also been used in literature [14]. This method starts with linear cooling of a melt, then takes snapshots at regular intervals (different temperatures) that can be used as a starting point for extending the simulations at constant temperature. For all the cooling methods, we would expect the results to be similar if the cooling rate allows for sufficient sample equilibration [15].

\subsubsection{Fitting Range}

Next we investigate the sensitivity of $T_{g}$ to the temperature ranges used to fit the fluid and glassy branches of the density-temperature curves. We took 20 independent stepped cooling simulations at three cooling rates of $0.005,0.04$ and $0.9 \mathrm{~K} \mathrm{ps}^{-1}$. We defined the fitting range of the glassy branch from the minimum temperature of $100 \mathrm{~K}$ to $T_{1}$, and the fitting range of the fluid branch from $T_{2}$ up to the highest temperature of $500 \mathrm{~K}$. These branches are then fit with linear functions of the form

$$
\begin{gathered}
\rho_{\text {glass }}(T)=A_{1} T+B_{1} \\
\rho_{\text {fluid }}(T)=A_{2} T+B_{2}
\end{gathered}
$$

as shown in Fig. 1. The larger the fitting range, the more data points are included, which should improve the fit, until the range overlaps with $T_{g}$, which should be avoided.

The dependence of the gradients, $A_{1}$ and $A_{2}$, on $T_{1}$ and $T_{2}$, respectively, is shown in Fig. 5 for a cooling rate of $0.04 \mathrm{~K} \mathrm{ps}^{-1}$. For the fluid branch, shown in Fig. 5(b), the gradient decreases in magnitude as $T_{2}$ decreases (apart from the point at high $T_{2}$ that has a very larger error) and 
becomes approximately constant around 300$350 \mathrm{~K}$, before again decreasing in magnitude, indicating a crossover from fluid to glassy regimes. For the glassy branch (Fig. 5(a)), which is over a smaller temperature range, the gradient rapidly increases in magnitude as $T_{1}$ crosses over into the fluid branch. However, neither the fluid branch nor the glassy branch have a sizeable range where the gradient remains approximately constant, which is a consequence of the gradual change in gradient with temperature, as opposed to a sharp transition at $T_{g}$. A similar outcome can be found from the variation of $B_{1}$ and $B_{2}$ with fitting range, shown in Supporting Information.

Given that we cannot determine a specific fitting range from the density, we now look at the variation of $T_{g}$ with the fitting range. Our aim is to find a range that can be used for all independent simulations and gives the most reliable estimate for $T_{g}$. We note that if a wider range of cooling rates were used then the fitting range may need to be adjusted with cooling rate as $T_{g}$ decreases with slower cooling rates but here we have focused on finding a single range for all cooling rates. Fig. 6 shows the average $T_{g}$ for each combination of $T_{1}$ and $T_{2}$ at a cooling rate of $\Gamma=0.04 \mathrm{~K} \mathrm{ps}^{-1}$. For the fitting ranges to be considered valid, $T_{g}$ must lie between $T_{1}$ and $T_{2}$. The hatched area on Figure 6 indicates the region where this condition is not met. Similar graphs were produced for the slowest and fastest cooling rates of 0.005 and $0.9 \mathrm{~K} \mathrm{ps}^{-1}$, respectively, shown in Supporting Information. Based on all three cases, values of $T_{1}=180 \mathrm{~K}$ and $T_{2}=300 \mathrm{~K}$ were chosen, which satisfied the above condition and allowed for a reasonable range of data to be fit for each branch. Using this range, we calculated the thermal expansion coefficient of PE in the fluid phase and obtained a value of $(6.26$ $\pm 0.05) \times 10^{-4} \mathrm{~K} \mathrm{ps}^{-1}$, which is in reasonable agreement with experiment and previously calculations. More information is available in Supporting Information.

A key point highlighted by this analysis is the large variation in $T_{g}$ on the fitting range. In the most extreme case at a cooling rate of $0.9 \mathrm{~K} \mathrm{ps}^{-1}$
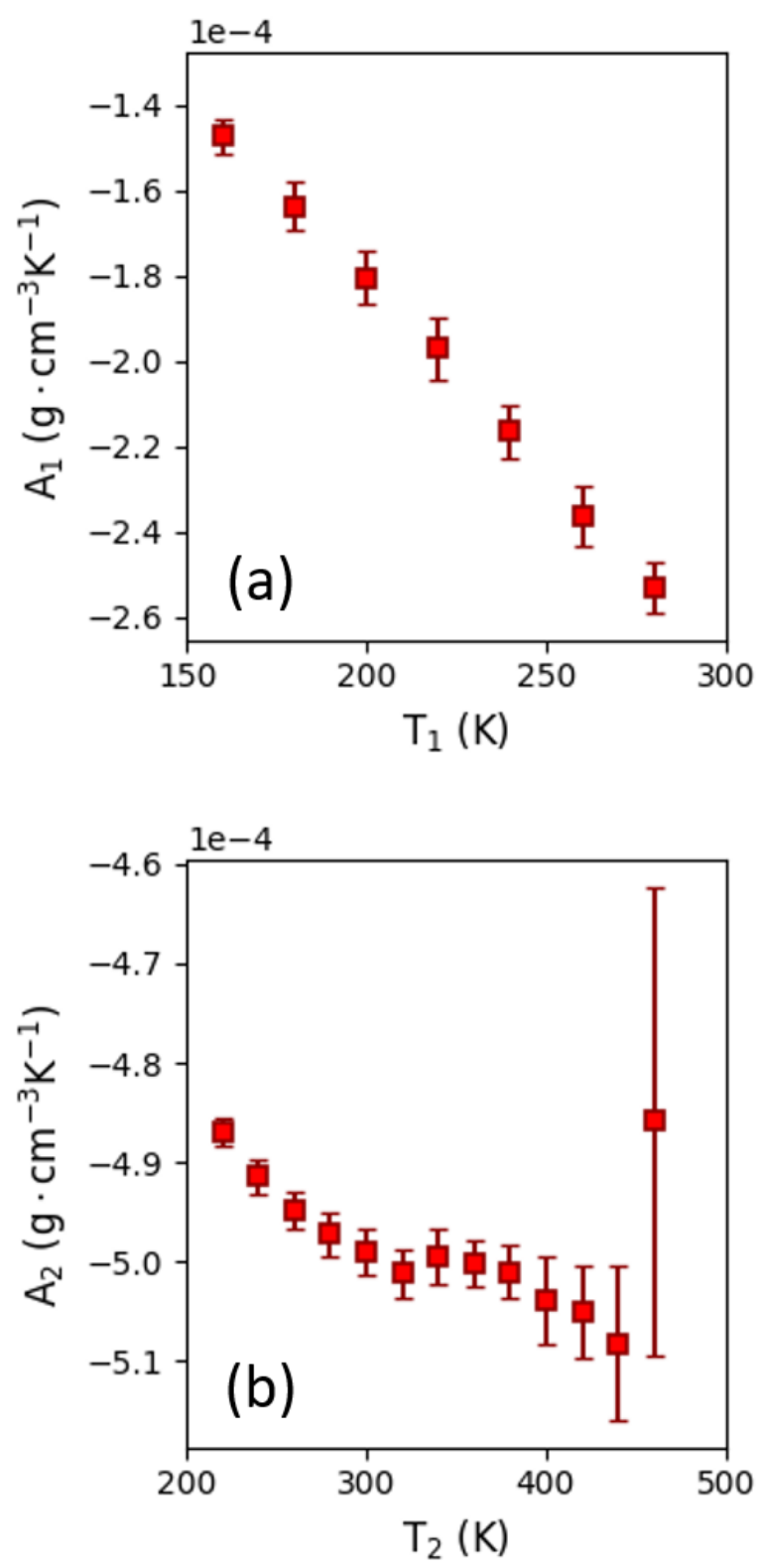

Figure 5: Dependence of the average gradients on fitting range for (a) the glassy and (b) the fluid branches using a cooling rate of $0.04 \mathrm{~K} \mathrm{ps}^{-1}$.

it was possible to obtain a $70 \mathrm{~K}$ variation in $T_{g}$ from the same set of 20 simulations, whilst still meeting the condition $T_{1}<T_{g}<T_{2}$ (see Supporting Information). $T_{g}$ was found to be particularly sensitive to $T_{1}$, the boundary for fitting the glassy branch, with $T_{g}$ consistently increasing with increasing $T_{1}$ for each cooling rate tested. An alternative technique used for fitting 


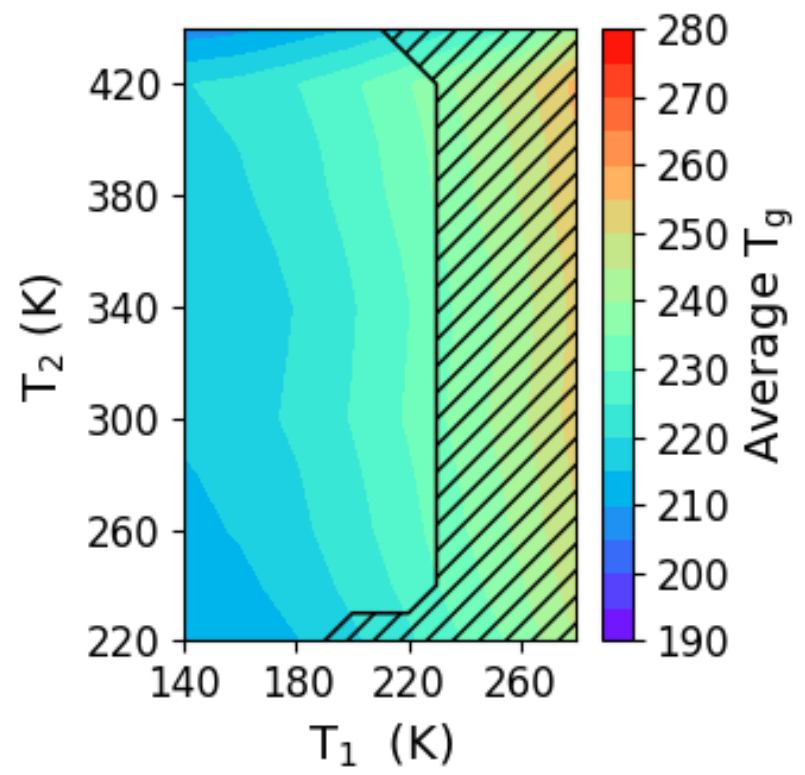

Figure 6: Average $T_{g}$ for varying values of $T_{1}$ and $T_{2}$ obtained at $\Gamma=0.04 \mathrm{~K} \mathrm{ps}^{-1}$. The black hatched area indicates a region where $T_{g}$ lies outside the range between $T_{1}$ and $T_{2}$.

the density vs temperature curves is to fit an hyperbola to the data and determine $T_{g}$ using either the asymtote tangent intersection or the coordinate of the hyperbola centre [27] and future work could compare the fitting techniques used here with the hyperbola method.

\subsection{Glass transition temperature for bulk PE and PE thin films}

The glass transition temperature is expected to vary with cooling rate as follows

$$
T_{g}(\Gamma)=T_{g 0}+\frac{A}{\ln (B \Gamma)}
$$

where $T_{g 0}$ is the glass transition temperature at infinitely low cooling rates, $\Gamma$ is the cooling rate and $A$ and $B$ are fitting parameters [28]. Further discussion about the dependence of $T_{g}$ on cooling rate can be found in Buchholz et al [15]. Fig. 7 shows the results obtained for $T_{g}$ vs cooling rate for the $\mathrm{PE}$ bulk and film with curves fit to equation (2).

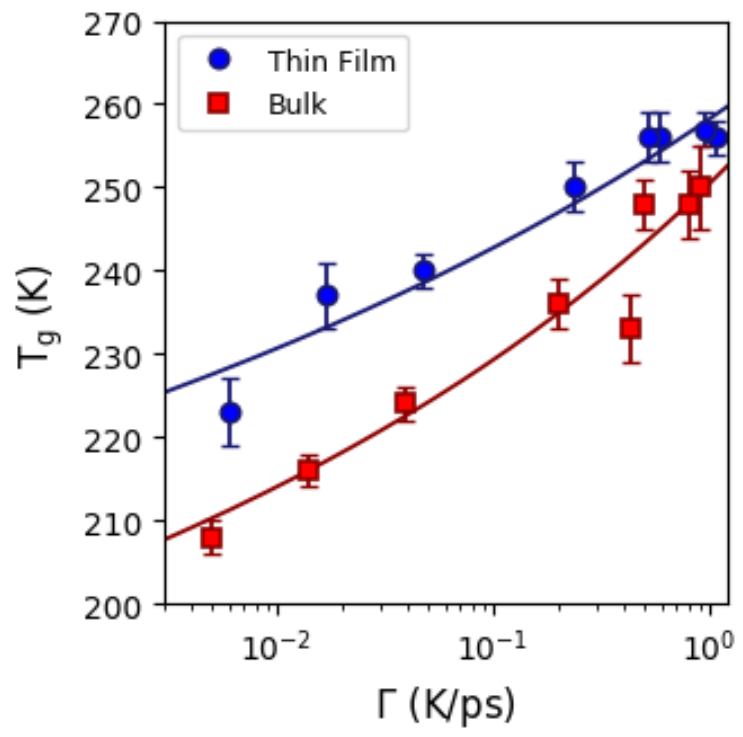

Figure 7: $T_{g}$ vs $\Gamma$ for $\mathrm{PE}$ in bulk (blue circles) and thin film (red squares).

For bulk PE, we found $A=-1538 \mathrm{~K}, B=0.07 \times$ $10^{-4} \mathrm{~K} \mathrm{ps}^{-1}$ and $T_{g}=120 \mathrm{~K}$. Ramos et al obtained fitting parameters of $A=-368.7 \mathrm{~K}$, $B=230 \times 10^{-4} \mathrm{~K} \mathrm{ps}^{-1}$ and $T_{g 0}=214 \mathrm{~K}$. They noted quantitative agreement between their value for $T_{g 0}$ and the value of $T_{g 0}=215 \mathrm{~K} \mathrm{ob}-$ tained by Yi et al [29]. While our obtained $T_{g} 0$ is significantly lower than those reported by $\mathrm{Yi}$ and Ramos, we note that Yi et al used a linear fit to obtain this value, and their individual values of $T_{g}$ are closer to those obtained in this work. Yang et al obtained a slightly lower $T_{g}$ of $200 \mathrm{~K}$ using a single cooling simulation at a cooling rate of $0.05 \mathrm{~K} \mathrm{ps}^{-1}$ [30], for a system of two 300 monomer chains and using a different force field. Simulation predictions for $T_{g}$ are shown in Supporting Information.

Experimental measurements of $T_{g}$ have varied significantly in the literature, partially due to difficulties with separating the glass transition from crystallisation [31]. Measured values for $T_{g}$ include $231 \pm 9 \mathrm{~K}$ [32], 190-200 K [33], $237 \mathrm{~K}$ [34], and $253 \mathrm{~K}$ [35]. Our simulations are performed at very high cooling rates where PE stays in an amorphous state. Our $T_{g}$ values at these high cooling rates are within the experimental range, 
however, our predicted $T_{g 0}$, which is more representative of experimental cooling rates, is much lower than experimental values of $T_{g}$. While we expect $T_{g 0}$ from simulations of short polymer chains to be lower than experimental $T_{g} 0$ measurements of high molecular weight polymers, we note that there is a large uncertainty in the fit over cooling rates due to statistical variation.

We now compare the $T_{g}$ of the $9 \mathrm{~nm}$ thin film under confinement between graphene layers to the bulk value. In order to ensure that the chosen fitting range would be suitable for the PE thin film, a similar analysis, as described in section 3.2.3, was performed on $\mathrm{PE}$ thin films at cooling rates of 1.07, 0.05 and $0.006 \mathrm{~K} \mathrm{ps}^{-1}$ and is shown in Supporting Information. The $9 \mathrm{~nm}$ confined PE film shows a clear increase in $T_{g}$ compared to the bulk, approximately $15 \mathrm{~K}$ higher for slower cooling rates, but then only slightly higher for faster cooling. This variation is likely due to poorer statistics associated with the faster cooling simulations. This is in qualitative agreement with a previous simulation study of polystyrene that found an increase of $T_{g}$ near a solid surface [8]. For the PE film the fit to equation (2) gave $A=-2036 \mathrm{~K}, B=0.0008 \times 10^{-4} \mathrm{~K} \mathrm{ps}^{-1}$ and $T_{g 0}=133 \mathrm{~K}$. The increase in $T_{g 0}$ is $13 \mathrm{~K}$, which is similar to the difference in $T_{g}$ at the slower cooling rates simulated here.

There are several issues that affect our calculation of $T_{g}$ and $T_{g 0}$. First, we note that the value of $T_{g 0}$ obtained here is very sensitive to statistical variation, with small changes in the values of $T_{g}$ resulting in significant changes in $T_{g 0}$. The fit could be improved with additional simulations at each cooling rate, and simulations at even slower cooling rates. Second, the shift in $T_{g}$ from bulk to the confined film will also depend on the nature of the surface. Our model uses a graphene layer, modelled by fixed uncharged atoms, and this may not represent quantitatively the PE-graphene interaction. This could be improved using a hierarchical modelling approach that uses quantum simulations to parameterise the PE-graphene force field parameters, similar to the development of an interface potential for polystyrene on gold [11]. In addition, a graphene surface will enhance crystallisation of PE, as observed for an adsorbed PE chain [36]. Crystallisation is not observed in the current simulations due to the very high cooling rates but would be an important consideration in slower cooling and in experimental measurements. Third, the $T_{g}$ shift also is specific to a $9 \mathrm{~nm}$ confined film and would vary quantitively with film width. Further work could focus on determining the $T_{g}$ interphase width, similar to the layer analysis approach of Hudzinskyy et al [8].

\section{Conclusions}

The polymer glass transition is an important property for many applications. MD simulations can provide insight into how an interface affects the glass transition temperature, however, studies rarely discuss the methodological details used to calculate $T_{g}$. In this work we have carried out a careful analysis of the impacts of statistical variation, cooling method and fitting range on the $T_{g}$ obtained from MD simulations and applied this to investigate how $T_{g}$ of $\mathrm{PE}$ changes when a thin film is confined between graphene surfaces.

From our results it is clear that multiple independent simulations should be performed at each cooling rate investigated, as independent simulations result in a significant variation in $T_{g}$. For example, at a cooling rate of $0.9 \mathrm{~K} \mathrm{ps}^{-1}, 100$ simulations gave an average $T_{g}$ of $238 \mathrm{~K}$, and a standard deviation of $22 \mathrm{~K}$. The choice of cooling method (stepped vs linear) has little impact on $T_{g}$. However, the fitting ranges used have a significant impact and for extreme cases can result in a difference of up to $70 \mathrm{~K}$ in the average $T_{g}$ from a sample of 20 independent simulations. This demonstrates the significance dependence on the statistics and fitting, and why it is of utmost importance to report this information in simulation studies of the glass transition.

We applied a consistent methodology for investigation of the impact of graphene surface on the 
glass transition temperature of PE. For a $9 \mathrm{~nm}$ thick film confined between graphene surfaces, the $T_{g}$ increased, compared to bulk, across all cooling rates tested, with an increase in $T_{g} 0$ of $13 \mathrm{~K}$. However, we note that the fitting to cooling rate is very sensitive to statistical fluctuations. In summary, we note that calculations of $T_{g}$ should perform a high number of independent MD simulations and that significant care should be taken in analysis and interpretation of the results.

\section{Acknowledgements}

Results were obtained using the ARCHIEWeSt High Performance Computer (www.archie-west.ac.uk) EPSRC grant number EP/K000586/1. David McKechnie was supported by an EPSRC Institutional Sponsorship Award (Grant Ref: $\mathrm{EP} / \mathrm{P} 511420 / 1)$. Jordan Cree received funding from CCP5 (www.ccp5.ac.uk) for a vacation bursary. We thank Peter Mills at Solvay Composites Group, U.K. for useful discussions. LAMMPS input files are available at https://doi.org/10.15129/fb75a450-ee45-4b39ac14-192ea5365a5d.

\section{References}

[1] Friedrich Kremer, Martin Tress, and Emmanuel U. Mapesa. Glassy dynamics and glass transition in nanometric layers and films: A silver lining on the horizon. J. Noncrystalline Solids, 407:277-283, 2015.

[2] Simone Napolitano, Emmanouil Glynos, and Nicholas B Tito. Glass transition of polymers in bulk, confined geometries, and near interfaces. Reports on Progress in Physics, 80(3):036602, jan 2017.

[3] J.-L. Barrat, J. Baschnagel, and A. Lyulin. J. Non-Crystalline Solids, 407:277-283, 2010 .
[4] A. R. C. Baljon, S. Williams, N. K. Balabaev, F. Paans, D. Hudzinskyy, and A. V. Lyulin. Simulated Glass Transition in FreeStanding Thin Polystyrene Films. J. Polymer Sci. B: Polymer Physics, 48:1160-1167, 2010 .

[5] Alexey V. Lyulin, Nikolay K. Balabaev, Arlette R. C. Baljon, Gerardo Mendoza, Curtis W. Frank, and Do Y. Yoon. Interfacial and topological effects on the glass transition in free-standing polystyrene films. $J$. Phys. Chem., 146:203314, 2017.

[6] Hiroshi Morita, Keiji Tanaka, Tisato Kajiyama, Toshio Nishi, and Masao Doi. Study of the Glass Transition Temperature of Polymer Surface by Coarse-Grained Molecular Dynamics Simulation. Macromolecules, 39:6233-6237, 2006.

[7] J. A Torres, P. F. Nealey, and J. J. de Pablo. Molecular Simulation of Ultrathin Polymeric Films near the Glass Transition. Phys. Rev. Lett., 85:3221, 2000.

[8] Dmytro Hudzinskyy, Alexey V. Lyulin, Arlette R. C. Baljon, Nikolaj K. Balabaev, and Mattias A. J. Michels. Effects of Strong Confinement on the GlassTransition Termperature in Simulated Atactic Polystyrene Films. Macromolecules, 44:2299, 2011.

[9] A. De Virgilis, A. Milchev, V. G. Rostiashvili, and T. A. Vilgis. Structure and dynamics of a polymer melt at an attractive surface. Eur. Phys. J. E, 35:97, 2012.

[10] Karen Johnston, Risto M. Nieminen, and Kurt Kremer. Hierarchical dualscale study of bisphenol-A-polycarbonate on a silicon surface: structure, dynamics and impurity diffusion. Soft Matter, 7:6457-6466, 2011.

[11] Karen Johnston and Vagelis Harmandaris. Properties of Short Polystyrene Chains Confined Between two Gold Surfaces Through a Combined Density Functional Theory and Classical Molecular Dynamics Approach. Soft Matter, 8:6320-6332, 2012. 
[12] Anastassia N. Rissanou, Albert John Power, and Vagelis Harmandaris. Structural and Dynamical Properties of Polyethylene/Graphene Nanocomposites through Molecular Dynamics Simulations. Polymers, 7:390-417, 2015.

[13] Alexey V. Lyulin, Nikolaj K. Balabaev, and M. A. J. Michels. Molecular-Weight and Cooling-Rate Dependence of Simulated $T_{g}$ for Amorphous Polystyrene. Macromolecules, 36:8574-8575, 2003.

[14] Javier Ramos, Juan F. Vega, and Javier Martinez-Salazar. Molecular dynamics simulations for the description of experimental molecular conformation, melt dynamics, and phase transitions in polyethylene. Macromolecules, 48:5016-5027, 2015.

[15] J. Buchholz, W. Paul, F. Varnik, and K. Binder. Cooling rate dependence of the glass transition temperature of polymer melts: Molecular dynamics. J. Chem. Phys., 117:7364-7372, 2002.

[16] Wolfgang Paul, Do Y. Yoon, and Grant D. Smith. An optimized united atom model for simulations of polymethylene melts. The Journal of Chemical Physics, 103:1702, 1995.

[17] Jaeeon Chang, Jie Han, Liu Yang, Richard L Jaffe, and Do Y Yoon. Structure and properties of polymethylene melt surfaces from molecular dynamics simulations. The Journal of Chemical Physics, 115(6):2831-2840, 2001.

[18] Wendy D. Cornell et al. A second generation force field for the simulation of proteins, nucleic acids and organic molecules. Journal of the American Chemical Society, 117:5179, 1995.

[19] Steve Plimpton. Fast parallel algorithms for short-range molecular dynamics. Journal of Computational Physics, 117(1):1-19, 1995.

[20] Kahyun Hur et al. Chain dynamics of ring and linear polyethylene melts from molecu- lar dynamics simulations. Macromolecules, 44:2311-2315, 2011.

[21] I. A. Bitsanis and G. Hatziioannou. Molecular dynamics simulations of the structure and dynamics of confined polymer melts. $J$. Chem. Phys., 92:3827, 1990.

[22] Kostas Ch. Daoulas, Vagelis A. Harmandaris, and Vlasis G. Mavrantzas. Detailed Atomistic Simulation of a Polymer Melt/Solid Interface: Structure, Density and Conformation of a Thin Film of Polyethylene Melt Adsorbed on Graphite. Macromolecules, 38:5780, 2005.

[23] L. Yelash, P. Virnau, K. Binder, and W. Paul. Slow process in confined polymer melts: Layer exchange dynamics at a polymer solid interface. Phys. Rev. E, 82:050801, 2010 .

[24] Anastassia N. Rissanou and Vagelis Harmandaris. Dynamics of various polymergraphene interfacial systems through atomistic molecular dynamics simulations. Soft Matter, 10:2876, 2014.

[25] D. V. Guseva, P. V. Komarov, and Alexey V. Lyulin. Molecular-dynamics simulations of thin polyisoprene films confined between amorphous silica substrates. $J$. Chem. Phys., 140:114903, 2014.

[26] Karen Johnston and Vagelis Harmandaris. Hierarchical multiscale modeling of polymer-solid interfaces: atomistic to coarse-grained description, and structural and conformational properties of polystyrene-gold systems. Macromolecules, 46:5741, 2013.

[27] Paul N. Patrone, Andrew Dienstfrey, Andrea R. Browning, Samuel Tucker, and Stephen Christensen. Uncertainty quantification in molecular dynamics studies of the glass transition temperature. Polymer, $87: 246-259,2016$.

[28] Katharina Vollmayr, Walter Kob, and Kurt Binder. How do the properties of a glass depend on the cooling rate? a computer simu- 
lation study of a lennard-jones system. The Journal of chemical physics, 105(11):47144728, 1996.

[29] Peng Yi, C Rebecca Locker, and Gregory C Rutledge. Molecular dynamics simulation of homogeneous crystal nucleation in polyethylene. Macromolecules, 46(11):4723-4733, 2013.

[30] Qing Yang, Xin Chen, Zhouwen He, Fengtao Lan, and Hui Liu. The glass transition temperature measurements of polyethylene: determined by using molecular dynamic method. RSC Adv., 6(12053), 2016.

[31] Umesh Gaur and Bernhard Wunderlich. The Glass Transition Temperature of Polyethylene. Macromolecules, 13:445-446, 1980.

[32] G. T. Davis and R. K. Eby. Glass transition of polyethylene: Volume relaxation. $J$. Appl. Phys., 44:4274-4281, 1973.

[33] J. M. G. Cowie and I. J. McEwen. Molecular Relaxations in Partially Hydrogenated cis- 1,4-Polybutadienes. A Guide to the Glass Transition Temperature of Amorphous Polyethylene. Macromolecules, 10:1124-1128, 1977.

[34] Umesh Gaur and Bernhard Wunderlich. Heat capacity and other thermodynamic properties of linear macromolecules. II. Polyethylene. J. Phys. Chem. Ref. Data, 10:119-152, 1981.

[35] G. Reiter and P. Kindl. Positron Lifetime Investigations on Linear Polyethylene Compared to Branched Polyethylene. Phys. Stat. Sol., 118:161-168, 1990.

[36] Max Gulde, Anastassia N. Rissanou, Vagelis Harmandaris, Marcus Müller, Sascha Schäfer, and Claus Ropers. Dynamics and Structure of Monolayer Polymer Crystallites on Graphene. Nano Letters, 16:69947000, 2016. 